\title{
Neuroprotection mediated by changes in the endothelial actin cytoskeleton
}

\author{
Ulrich Laufs, ${ }^{1,2}$ Matthias Endres, ${ }^{3}$ Nancy Stagliano, ${ }^{4}$ Sepideh Amin-Hanjani, ${ }^{3}$ \\ Dao-Shan Chui, ${ }^{1}$ Shui-Xiang Yang, ${ }^{1}$ Tommaso Simoncini, ${ }^{1}$ Masaru Yamada, ${ }^{3}$ \\ Elena Rabkin, ${ }^{5}$ Philip G. Allen, ${ }^{6}$ Paul L. Huang, ${ }^{4}$ Michael Böhm, ${ }^{2}$ Frederick J. Schoen, ${ }^{5}$ \\ Michael A. Moskowitz, ${ }^{3}$ and James K. Liao ${ }^{1}$
}

${ }^{1}$ Cardiovascular Division, Department of Medicine, Brigham and Women's Hospital and Harvard Medical School, Boston, Massachusetts, USA

${ }^{2}$ Klinik III für Innere Medizin, Universität zu Köln, Köln, Germany

${ }^{3}$ Stroke and Neurovascular Regulation Laboratory, and

${ }^{4}$ Cardiovascular Research Center, Massachusetts General Hospital, Charlestown, Massachusetts, USA

${ }^{5}$ Department of Pathology, Brigham and Women's Hospital, Boston, Massachusetts, USA

${ }^{6}$ Experimental Medicine Division, Brigham and Women's Hospital, Boston, Massachusetts, USA

Address correspondence to: James K. Liao, Brigham and Women's Hospital, 221 Longwood Avenue, LMRC-322, Boston, Massachusetts 02115, USA. Phone: (617) 732-6538; Fax: (617) 264-6336; E-mail: jliao@rics.bwh.harvard.edu.

Ulrich Laufs and Matthias Endres contributed equally to this work.

Received for publication February 16, 2000, and accepted in revised form May 31, 2000.

Cerebral blood flow is regulated by endothelium-derived nitric oxide (NO), and endothelial NO synthase-deficient (eNOS-deficient; $e \mathrm{NOS}^{-/-}$) mice develop larger cerebral infarctions following middle cerebral artery (MCA) occlusion. We report that disruption of Rho-mediated endothelial actin cytoskeleton leads to the upregulation of eNOS expression and reduces the severity of cerebral ischemia following MCA occlusion. Mice treated with the Rho inhibitor Clostridium botulinum C3 transferase $(10 \mu \mathrm{g} / \mathrm{d})$ or the actin cytoskeleton disrupter cytochalasin $\mathrm{D}(1 \mathrm{mg} / \mathrm{kg})$ showed a two- to fourfold increase in vascular eNOS expression and activity. This increase in eNOS expression was not due to increases in eNOS gene transcription, but to prolongation of eNOS mRNA half-life from 10 \pm 3 hours to $24 \pm 4$ hours. Indeed, endothelial cells overexpressing a dominant-negative Rho mutant (N19RhoA) exhibited decreased actin stress fiber formation and increased eNOS expression. Inhibition of vascular Rho guanosine-5'-triphosphate binding activity by the 3-hydroxy-3-methylglutarylcoenzyme A reductase inhibitor simvastatin increased cerebral blood flow to ischemic regions of the brain, and mice treated with simvastatin, C3 transferase, or cytochalasin D showed smaller cerebral infarctions following MCA occlusion. No neuroprotection was observed with these agents in $e \mathrm{NOS}^{-/-}$ mice. These findings suggest that therapies which target the endothelial actin cytoskeleton may have beneficial effects in ischemic stroke.

J. Clin. Invest. 106:15-24 (2000).

\section{Introduction}

Ischemic stroke remains a leading cause of mortality and morbidity worldwide. Current treatments for ischemic stroke are limited to agents that block platelet aggregation or the coagulation cascade but that do not directly affect blood flow during cerebral ischemia. Cerebral vascular tone and blood flow are regulated by endothelium-derived nitric oxide (NO) (1), and endothelial NO synthase-deficient (eNOS-deficient; $e \mathrm{NOS}^{-/-}$) mice develop larger cerebral infarctions following middle cerebral artery (MCA) occlusion (2). The loss of endothelium-derived NO impairs vascular function in part by promoting vasoconstriction (3), platelet aggregation (4), smooth muscle cell proliferation (5), and leukocyte adhesion (6). Mice which lack the gene for eNOS have higher blood pressure (7) and are prone to larger cerebral infarctions following MCA occlusion (2). Similarly, inhibition of eNOS activity by L-nitroargi- nine methylester (L-NAME) results in a decrease in cerebral blood flow and an increase in infarct size after focal ischemia (8). In contrast, enhanced endothelial NO production by administration of 3-hydroxy-3-methylglutaryl-coenzyme A (HMG-CoA) reductase inhibitors or statins, which upregulate eNOS expression (9), or by infusion of the substrate for eNOS, L-arginine, which increases eNOS activity (10), has been shown to decrease the total cerebral tissue at risk after vascular occlusion, probably by enhancing collateral blood flow in the compromised brain (11). Thus, factors or conditions which favor endothelial NO production may be beneficial in limiting the severity of cerebral ischemia.

The endothelial actin cytoskeleton and eNOS activity are coregulated by diverse stimuli such as hormones (12), growth factors (13), and laminar flow (14). It is not known, however, whether changes in the endothelial actin cytoskeleton affect eNOS expression and 
activity. Changes in the actin cytoskeleton are mediated, in part, by the small guanosine- $5^{\prime}$-triphosphate binding (GTP-binding) protein Rho (15). We recently reported that Rho negatively regulates the eNOS expression in endothelial cell culture $(9,16)$, but the beneficial effects of Rho inhibition in vivo have not been demonstrated. Furthermore, it is not known whether the upregulation of eNOS by Rho inhibition is mediated by the actin cytoskeleton or some other downstream target of Rho. The purpose of this study, therefore, was to determine whether inhibition of the endothelial actin cytoskeleton can produce NO-mediated neuroprotection in ischemic stroke.

\section{Methods}

Reagents. All standard culture reagents were obtained from JRH Biosciences (Lenexa, Kansas, USA). Simvastatin tablets were obtained commercially and chemically activated by alkaline hydrolysis prior to use as previously described (17). Cytochalasin D and nocodazole were purchased from Calbiochem-Novabiochem Corp. (San Diego, California, USA). [ $\left.{ }^{32} \mathrm{P}\right] \mathrm{CTP}(3000 \mathrm{Ci} / \mathrm{mmol})$ and $\left[{ }^{35} \mathrm{~S}\right] \mathrm{GTP} \gamma \mathrm{S}(1250 \mathrm{Ci} / \mathrm{mmol})$ were supplied by New England Nuclear (Boston, Massachusetts, USA). The antibody detection kit (Enhanced Chemiluminescence) and the nylon nucleic acid (Hybond) and protein (PVDF) transfer membranes were purchased from Amersham Life Sciences Inc. (Arlington Heights, Illinois, USA). The Clostridium botulinum C3 transferase was purchased from List Biological Laboratories Inc. (Campbell, California, USA). C3 transferase was supplied as lyophilized powder and was reconstituted with sterile phosphate buffered saline $(1 \mu \mathrm{g} / \mu \mathrm{L})$.

Experimental protocol. All animal experiments were conducted in accordance with National Institutes of Health, Brigham and Women's Hospital, and Massachusetts General Hospital institutional guidelines. SV/129C57BL/6 male mice (18-22 g; Taconic Farm, Germantown, New York, USA) (i.e., littermates for $\mathrm{NOS}^{-/-}$mice) (7) were injected subcutaneously with $0.1 \mathrm{~mL}$ of activated simvastatin $(20 \mathrm{mg} / \mathrm{kg} / \mathrm{d})$ or a corresponding volume of phosphate buffered saline once daily for 14 days. C3 transferase $(10 \mu \mathrm{g} / \mathrm{d}$ for 14 days) was infused via miniosmotic pumps (ALZET model 2002 osmotic pump; ALZA Corp., Mountain View, California, USA), which were implanted subcutaneously. Control mice were infused with normal saline under otherwise identical experimental conditions. Cytochalasin D (0.1 and 1.0 $\mathrm{mg} / \mathrm{kg} / \mathrm{d}$ ) or saline was injected intraperitoneally.

Model of focal cerebral ischemia. Animals were anesthetized with $1.5 \%$ halothane and maintained on $1.0 \%$ halothane in $70 \% \mathrm{~N}_{2} \mathrm{O}$ and $30 \% \mathrm{O}_{2}$ by a face mask. Cerebral infarcts were produced by 2 hours of MCA occlusion followed by reperfusion. A silicone-coated 8-0 monofilament was introduced into the internal carotid artery and advanced to occlude the MCA. After 2 hours, the animals were briefly re-anesthetized and the filament withdrawn. Relative cerebral blood flow was measured by Laser-Doppler flowmetry (PF2B; Perimed,
Stockholm, Sweden) $(9,18)$. In randomly selected animals, the left femoral artery was cannulated with a PE10 catheter for arterial blood pressure and blood gas determination as described (9). Arterial blood samples were analyzed for $\mathrm{pH}$, arterial oxygen pressure, and partial pressure of carbon dioxide using a blood gas $/ \mathrm{pH}$ analyzer (Corning 178; Ciba-Corning Diagnostics Corp., Medford, Massachusetts, USA). Rectal temperature was monitored and maintained by means of a feedback temperature control unit (Frederick Haer and Co., Brunswick, Maine, USA).

Cerebral blood flow. Six hours following MCA occlusion, halothane-anesthetized mice were administered $\left[{ }^{14} \mathrm{C}\right]$-iodoantipyrine $(5 \mu \mathrm{Ci}$ in $100 \mu \mathrm{L}$ saline) for 1 minute by steady infusion pump. Arterial blood samples were collected at 5-second intervals onto preweighed filter paper disks and measured by liquid scintillation spectrometry (RackBeta 1209; PharmaciaWallac, Gaithersburg, Maryland, USA). Immediately after decapitation, the whole head was immersed in chilled isopentane over dry ice $\left(-45^{\circ} \mathrm{C}\right)$, and then the frozen brains were sectioned coronally $(20 \mu \mathrm{m})$. The sections were thaw-mounted on glass coverslips, dried $\left(60^{\circ} \mathrm{C}\right)$, and exposed for 3 days to Kodak SB-5 autoradiographic film along with $\left[{ }^{14} \mathrm{C}\right]$-polymer standards (Amersham Life Sciences Inc.).

Cerebral infarct size. Twenty-two hours after reperfusion, mice were sacrificed and brains were removed. The brains were divided into five coronal $2-\mathrm{mm}$ sections using a mouse brain matrix (RBM-2000C; Activational Systems, Warren, Michigan, USA), stained in a 2\% 2,3,5-triphenyltetrazolium chloride solution in normal saline, and fixed in a $10 \%$ formalin solution overnight. Infarction volume was quantitated using a computer image analysis system (M4; Imaging Research Inc., St. Catherine's, Ontario, Canada) and calculated by adding the volumes of each section directly as described $(9,18)$.

Cell cultures. Human endothelial cells were harvested enzymatically with type II collagenase (Worthington Biochemical Corp., Lakewood, New Jersey, USA) as described $(16,17)$ and were maintained in Medium 199 (GIBCO BRL, Gaithersburg, Maryland, USA), containing HEPES $(25 \mathrm{mmol} / \mathrm{L})$, heparin $(50 \mathrm{U} / \mathrm{mL})$, endothelial cell growth factor $(50 \mu \mathrm{g} / \mathrm{mL})$, L-glutamine $(2$ $\mathrm{mmol} / \mathrm{L}$ ), antibiotics, and $5 \%$ fetal bovine serum.

Immunobistochemistry. Mice treated with the indicated conditions were sacrificed and the aortas were perfusion fixed (at $80 \mathrm{mmHg}$ ) with $3.7 \%$ formaldehyde in PBS. Approximately 5- to $10-\mathrm{mm}$ lengths of aorta were washed with PBS, transversely sectioned, and stained with a monoclonal anti-eNOS antibody (1:100 dilution; Transduction Laboratories, Lexington, Kentucky, USA). As a control, vehicle-treated aortas were stained with a pre-immune IgG or monoclonal anti-CD31 antibody (Vector Laboratories, Burlingame, California, USA). All samples were reviewed by two blinded observers. The entire circumference of the vessel wall, consisting of approximately 85-90 endothelial cells for 
each condition, was examined. The number of endothelial cells did not vary with the treatment conditions. The blinded observers rated each vessel for eNOS staining based upon the following grading criteria: grade 0: no staining; grade 1: weak staining in $<50 \%$ of cells; grade 2 : weak staining in $>50 \%$ of cells or strong staining in $<10 \%$ of cells; grade 3 : strong staining in $10-50 \%$ of cells; grade 4 : strong staining in $>50 \%$ of cells; and grade 5: strong staining in $100 \%$ of cells.

Immunofluorescence. Bovine aortic endothelial cells (60-70\% confluent) were transfected with $5 \mu \mathrm{g}$ of the indicated RhoA, Rac-1, and Cdc42 cDNA mutant constructs using Lipofectamine (GIBCO BRL, Grand Island, New York, USA). All constructs contained an $\mathrm{NH}_{2}$-terminal c-myc tag. Approximately 48 hours after transfection, cells were fixed with $3.7 \%$ formaldehyde in PBS and permeabilized with $0.1 \%$ Triton X-100 (Sigma Chemical Co., St. Louis, Missouri, USA) in PBS. For staining of actin stress fibers, cells were incubated with $1 \mu \mathrm{M}$ phalloidin (TRITC-labeled; Sigma Chemical Co.) for 30 minutes, washed, and incubated overnight with rabbit anti-c-myc antibody (1:50 in 1\% BSA; Santa Cruz Biotechnology Inc., Santa Cruz, California, USA). For staining of eNOS, cells were incubated overnight with goat anti-c-myc antibody (1:50 in 1\% BSA, Santa Cruz Biotechnology Inc.) and mouse anti-eNOS (1:50; Transduction Laboratories) at $4^{\circ} \mathrm{C}$. After washing with $1 \%$ BSA, TRITC-conjugated goat anti-mouse IgG (red fluorescence) and FITC-conjugated goat anti-rabbit IgG (green fluorescence) (Jackson ImmunoResearch Laboratories Inc., West Grove, Pennsylvania, USA) were used as secondary antibodies (1:500 in 1\% BSA) and incubated at $4^{\circ} \mathrm{C}$ for 3 hours in the dark. Cells were washed five times with PBS and mounted onto glass slides using the ProLong Antifade Kit (Molecular Probes Inc., Eugene, Oregon, USA). Immunofluorescence was visualized using an Olympus BX 60F inverted fluorescence microscope (Olympus America Inc., Lake Success, New York, USA). Photographic images were taken from five random fields.

eNOS activity assay. Mouse aortas were homogenized in ice-cold PBS containing $1 \mathrm{mM}$ EDTA using a polytron. The homogenates were pelleted in a microfuge $(2$ minutes, $13,800 \mathrm{~g}, 4^{\circ} \mathrm{C}$ ) to remove the insoluble material. Protein concentration was determined with the Micro BCA Assay Kit (Pierce Chemical Co., Rockford, Illinois, USA), and $5 \mu \mathrm{g}$ of protein extracts from each sample were used for the eNOS assay. The eNOS activity was detected by measuring the conversion of $\left[{ }^{3} \mathrm{H}\right] \mathrm{L}-$ arginine to $\left[{ }^{3} \mathrm{H}\right] \mathrm{L}$-citrulline at $37^{\circ} \mathrm{C}$ for 30 minutes with the eNOS assay kit (Calbiochem-Novabiochem Corp.) as described (9). Unlabeled L-arginine was added to $\left[{ }^{3} \mathrm{H}\right] \mathrm{L}$-arginine (specific activity $60 \mathrm{Ci} / \mathrm{mmol}$ ) at a ratio of $3: 1$. Rat cerebellum extracts, containing elevated amounts of neuronal NOS, were used as positive controls, while samples incubated in the presence of the competitive NOS inhibitor L-NAME $(1 \mathrm{mM})$ were used to determine nonspecific activity. Nonspecific activity accounted for $20-35 \%$ of total activity.
Competitive RT-PCR. Aortas were quickly isolated and frozen after sacrifice. Total RNA isolation, reverse transcription, and semiquantitative competitive PCR were performed according to standard techniques. Primer pairs for eNOS and GAPDH were used as described previously (9). Each PCR cycle consisted of denaturing at $94^{\circ} \mathrm{C}$ for 30 seconds, annealing at $60^{\circ} \mathrm{C}$ for $30 \mathrm{sec}-$ onds, and elongation at $72^{\circ} \mathrm{C}$ for 60 seconds. The linear exponential phases for eNOS and GAPDH PCR were 35 and 22 cycles, respectively. Equal amounts of corresponding NOS and GAPDH RT-PCR products were loaded on $1.5 \%$ agarose gels. Optical densities of ethidium bromide-stained DNA bands were quantitated and results were expressed as NOS/GAPDH ratios. For competitive RT-PCR, an additional 700 bp from Gs $\alpha$ (SacII-EcoRI fragment) was inserted into our eNOS and GAPDH PCR fragments, and these larger mutant eNOS and GAPDH fragments were used to compete with the corresponding reverse-transcribed eNOS or GAPDH from the samples. Increasing amounts of mutant eNOS ( $\triangle \mathrm{eNOS})$ or GAPDH $(\triangle \mathrm{GAPDH})$ template $\left(0.001\right.$ to $100 \mathrm{atmol} ; 1 \mathrm{atmol}=10^{-18}$ mole) were added to $2 \mu \mathrm{g}$ of reverse-transcribed mRNA derived from $1 \mathrm{mg}$ of murine aortic tissue. PCR using the same pair of primers yielded the expected two bands (i.e., wild-type and mutant fragments), which were confirmed by DNA sequencing.

Rho GTP-binding assay. Aortas were quickly isolated and snapped-frozen in isopentane on dry ice. Cell lysates were obtained by homogenization, and Rho
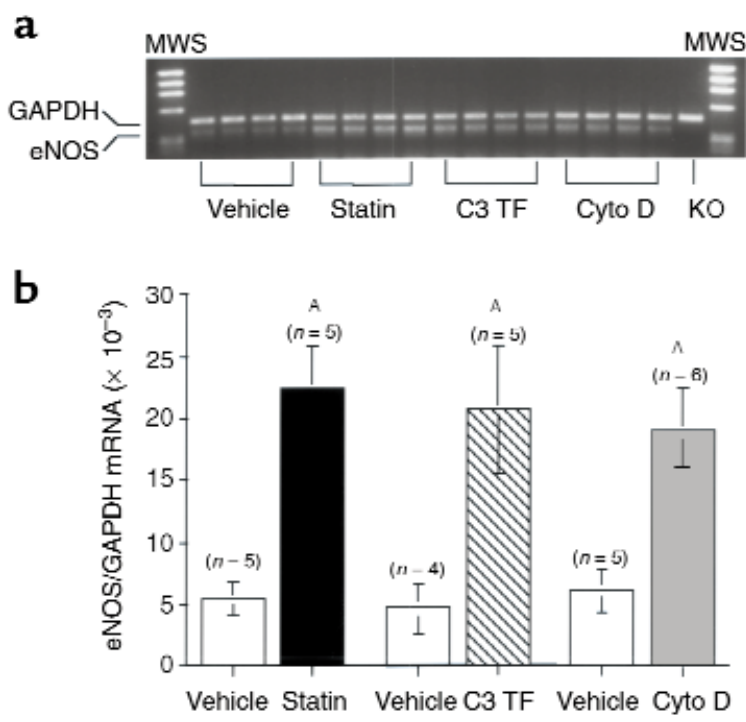

\section{Figure 1}

(a) RT-PCR of eNOS and GAPDH using aortic tissues from wild-type $\mathrm{SV} / 129$ or $\mathrm{NNOS}^{-1-}(\mathrm{KO})$ mice treated with saline (Vehicle), simvastatin (Statin, $20 \mathrm{mg} / \mathrm{kg} / \mathrm{d}$ subcutaneously for 14 days), C. botulinum C3 transferase (C3 TF, $10 \mu \mathrm{g} / \mathrm{d}$ subcutaneously for 14 days), and cytochalasin D (Cyto D, $1 \mathrm{mg} / \mathrm{kg}$ intraperitoneally for 24 hours). (b) Competitive RT-PCR showing the effects of statin, C3 TF, and cyto D on eNOS mRNA relative to GAPDH mRNA. The differences compared with treatment with vehicle were statistically significant $\left({ }^{A} P<\right.$ 0.05). MWS, molecular weight standard. 
a

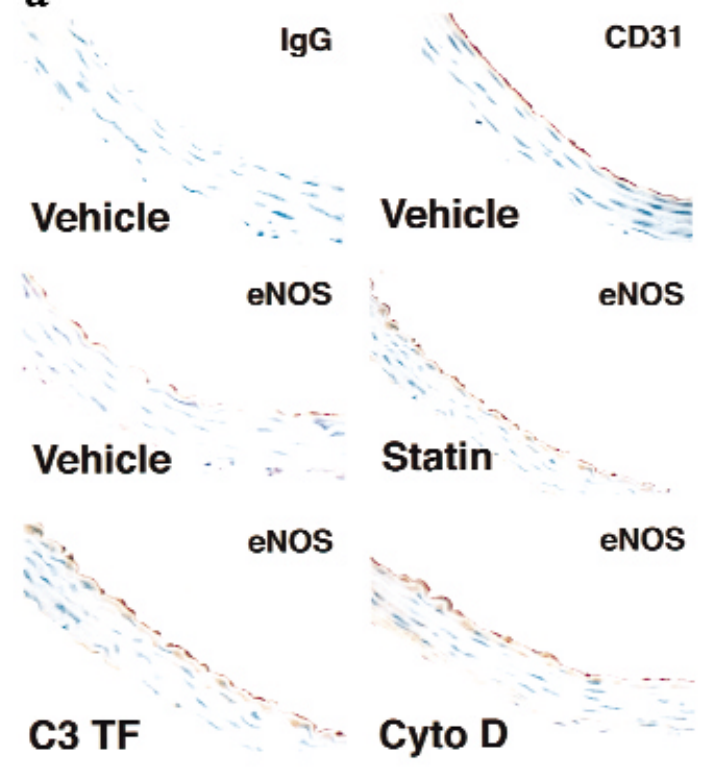

b

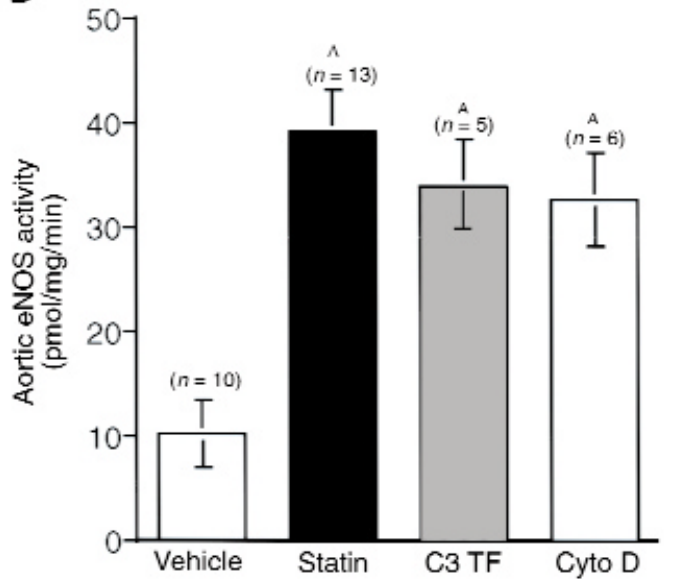

Figure 2

(a) Immunohistochemistry using pre-immune (IgG), CD31, or eNOS antibody, and (b) eNOS activity measured by [ $\left.{ }^{3} \mathrm{H}\right]$-arginine to $\left[{ }^{3} \mathrm{H}\right]-$ citrulline conversion in aorta from mice treated with statin, C3 TF, or cyto D (1 mg/kg). The differences between treatment with vehicle and treatment with statin, C3 TF, or cyto D were statistically significant $\left({ }^{A} P<0.05\right)$.

GTP-binding assay was performed using a Rho A-specific antibody and $\left[{ }^{35} \mathrm{~S}\right] \mathrm{GTP} \gamma \mathrm{S}$ as described (16).

\section{Results}

Disruption of the endothelial actin cytoskeleton upregulates eNOS. To determine the actin cytoskeletal regulation of eNOS, wild-type SV/129 mice were treated with preactivated simvastatin (statin; $20 \mathrm{mg} / \mathrm{kg} / \mathrm{d}$ subcutaneously for 14 days), C. botulinum C3 transferase $(10 \mu \mathrm{g} / \mathrm{d}$ subcutaneously for 14 days), or the actin cytoskeleton disrupter cytochalasin D $(0.1$ and $1 \mathrm{mg} / \mathrm{kg}$ intraperitoneally for 24 hours). Mice treated with statin, C3 transferase, or cytochalasin D were not different in terms of morphologic appearance, weight, or appetite. Treatment with statin, C3 transferase, or cytochalasin D increased aortic eNOS mRNA expression more than twofold compared with that of GAPDH (Figure 1a). Interestingly, a lower concentration of cytochalasin D $(0.1 \mathrm{mg} / \mathrm{kg})$ did not produce a significant increase in eNOS expression. By quantitative competitive RT-PCR, aortas from vehicle-treated mice had $0.032 \pm 0.008$ atmol of eNOS mRNA and $5.8 \pm 1.2$ atmol GAPDH mRNA per milligram of aortic tissue. Treatment with statin, C3 transferase, or cytochalasin D increased the amount of eNOS mRNA (i.e., standardized to the same amount of GAPDH in each sample) to $0.13 \pm 0.02,0.12$ \pm 0.03 , and $0.11 \pm 0.02 \mathrm{atmol} / \mathrm{mg}$ of aortic tissue, respectively. The increases in eNOS mRNA expression correlated with increases in aortic eNOS protein levels by immunohistochemistry as assessed by two blinded observers (Figure 2a). Using the scoring criteria described, the following semiquantitative results were obtained for eNOS staining: vehicle: $2.3 \pm 0.5$; statin: $4.3 \pm 0.3$; C3 transferase: $4.5 \pm 0.3$; and cytochalasin D: $4.0 \pm 0.5(P<0.05$ compared with vehicle for all conditions). This increase in eNOS protein expression correlated with a greater than threefold increase in aortic eNOS activity (Figure 2b).

To determine the mechanism of increased eNOS expression, we assessed eNOS gene transcription and mRNA stability in the presence of C3 transferase or cytochalasin D in vitro. Nuclear run-on assays showed that C3 transferase and cytochalasin D did not induce eNOS gene transcription in human vascular endothelial cells (Figure 3a). Induction of eNOS gene transcription, however, was observed following exposure to laminar shear stress (12 dynes/cm², 24 hours) (sixfold increase, $P<0.05)$. Similarly, when bovine aortic endothelial cells were transfected with a $-1.8 \mathrm{~kb}$ human eNOS promoter-luciferase reporter gene construct, neither C3 transferase nor cytochalasin D increased eNOS promoter activity (Figure 3b). In contrast, laminar shear stress increased eNOS promoter activity 13fold $(P<0.05)$. To determine whether the increase in eNOS expression was due to post-transcriptional mechanisms, endothelial cells were treated with C3 transferase or cytochalasin $\mathrm{D}$ in the presence or absence of the mRNA synthetase inhibitor 5,6-dichlorobenzimidazole riboside (DRB). Both C3 transferase and cytochalasin D prolonged eNOS mRNA half-life from $10 \pm 3$ to $24 \pm 4$ hours $(P<0.05$ for both) (Figures $3 c$ and $3 \mathrm{~d}$ ). These findings indicate that changes in the endothelial actin cytoskeleton affect eNOS expression primarily by altering eNOS mRNA stability. 
Rho as a regulator of the actin cytoskeleton and eNOS. To determine whether eNOS upregulation is specifically due to Rho-induced actin cytoskeletal changes, we overexpressed c-myc-tagged constitutively active or dominant-negative mutants of Rho family members RhoA, Rac-1, and Cdc42 in bovine aortic endothelial cells. Since transfection efficiency using the calcium-phosphate coimmunoprecipitation method is approximately $15 \%$, we examined the effects of these cDNA constructs on single cells using dual-labeled c-myc and eNOS immunofluorescence. Endothelial cells overexpressing constitutively active RhoA, Rac- 1 , and Cdc42 or dominant-negative Rac-1 and Cdc42 did not show changes in eNOS expression compared to nontransfected cells, although their respective actin cytoskeletons were substantially altered. In particular, cells overexpressing constitutively active Cdc42 appear larger than nontransfected cells (Figure 4). Only endothelial cells overexpressing dominant-negative RhoA (N19RhoA) showed substantially higher levels of eNOS expression compared with nontransfected cells.

The increases in the amount of actin stress fibers, assembly of focal adhesion complex, and reorganization of the cytoskeleton are mediated, in part, by Rho kinases and subsequent myosin light chain (MLC) phosphorylation $(15,19)$. To address the question of whether eNOS expression is related to the amount of actin stress fibers, we treated endothelial cells with agents that specifically disrupt the actin and microtubule cytoskeleton: cytochalasin $\mathrm{D}$ and nocodazole, respectively.
Cytochalasin D caused a concentration-dependent increase in eNOS expression (Figure 5a). This upregulation of eNOS was specific to changes in the actin cytoskeleton, since inhibition of the microtubule cytoskeleton by nocodazole decreased eNOS expression (Figure 5b). Compared with untreated cells, endothelial cells treated with C3 transferase or cytochalasin D showed changes in the actin cytoskeleton consistent with decreased actin stress fibers and focal adhesion complexes (Figure 5c). The HMG-CoA reductase inhibitor simvastatin inhibits Rho geranylgeranylation and thereby inhibits Rho membrane localization and activity (16). Overexpression of the dominant-negative N19RhoA construct also decreased the amount of endothelial actin stress fibers and, reciprocally, increased eNOS expression (Figures $5 \mathrm{c}$ and d). Inhibition of the microtubule cytoskeleton with nocodazole, however, produced an increase in actin stress fibers (data not shown). These findings agree with previous studies showing that the microtubule and actin cytoskeleton are counter-regulated (20).

Pharmacologic and genetic regulation of Rho activity and eNOS expression. The Rho GTP-binding activity in salinetreated (vehicle) mouse aorta was $560 \pm 85 \mathrm{fmol} / \mathrm{mg} / \mathrm{min}$ (Figure 6a). Treatment with simvastatin $(20 \mathrm{mg} / \mathrm{kg} / \mathrm{d}, 14$ days) or C3 transferase (10 $\mu \mathrm{g} / \mathrm{d}, 14$ days) decreased aortic Rho GTP-binding activity to $220 \pm 45$ and $340 \pm 65$ $\mathrm{fmol} / \mathrm{mg} / \mathrm{min}$, respectively ( $P<0.05$ for both) (Figure 6a). Treatment with cytochalasin $\mathrm{D}$, however, produced a twofold increase in Rho GTP-binding activity $(1180 \pm 230$

Table 1

Physiologic parameters before and after MCA occlusion

\begin{tabular}{|c|c|c|c|c|c|c|}
\hline & Vehicle & Statin & Vehicle & C3 TF & Vehicle & Cyto D \\
\hline \multicolumn{7}{|c|}{ MABP $(\mathrm{mmHg})$} \\
\hline Pre & $95 \pm 5$ & $97 \pm 5$ & $102 \pm 1$ & $100 \pm 1$ & $87 \pm 12$ & $93 \pm 10$ \\
\hline Post & $89 \pm 6$ & $92 \pm 4$ & $90 \pm 6$ & $92 \pm 3$ & $80 \pm 16$ & $88 \pm 12$ \\
\hline \multicolumn{7}{|c|}{ Core temperature $\left({ }^{\circ} \mathrm{C}\right)$} \\
\hline During & $36.6 \pm 0.3$ & $36.5 \pm 0.4$ & $36.4 \pm 0.2$ & $36.6 \pm 0.1$ & $36.9 \pm 0.1$ & $36.6 \pm 0.6$ \\
\hline \multicolumn{7}{|l|}{$\mathrm{pH}$} \\
\hline Pre & $7.31 \pm 0.03$ & $7.30 \pm 0.02$ & $7.33 \pm 0.02$ & $7.33 \pm 0.02$ & $7.30 \pm 0.01$ & $7.30 \pm 0.01$ \\
\hline Post & $7.28 \pm 0.03$ & $7.27 \pm 0.03$ & $7.28 \pm 0.02$ & $7.26 \pm 0.03$ & $7.30 \pm 0.01$ & $7.30 \pm 0.01$ \\
\hline \multicolumn{7}{|c|}{$\mathrm{PaO}_{2}(\mathrm{mmHg})$} \\
\hline Pre & $135 \pm 11$ & $133 \pm 12$ & $138 \pm 9$ & $133 \pm 12$ & $122 \pm 35$ & $165 \pm 21^{A}$ \\
\hline Post & $125 \pm 10$ & $131 \pm 5$ & $135 \pm 9$ & $139 \pm 5$ & $115 \pm 22$ & $128 \pm 16$ \\
\hline \multicolumn{7}{|c|}{$\mathrm{PaCO}_{2}(\mathrm{mmHg})$} \\
\hline Pre & $47.2 \pm 3.8$ & $47.5 \pm 2.0$ & $47.0 \pm 4.0$ & $46.0 \pm 2.0$ & $48.5 \pm 1.0$ & $49.9 \pm 3.7$ \\
\hline Post & $47.2 \pm 2.4$ & $46.8 \pm 1.5$ & $47.0 \pm 2.0$ & $46.0 \pm 1.0$ & $47.8 \pm 4.4$ & $46.9 \pm 4.3$ \\
\hline \multicolumn{7}{|c|}{ rCBF (\%) } \\
\hline Pre & $14 \pm 3$ & $14 \pm 2$ & $15 \pm 3$ & $13 \pm 2$ & $11 \pm 4$ & $17 \pm 9$ \\
\hline Post & $105 \pm 11$ & $95 \pm 8$ & $98 \pm 7$ & $99 \pm 6$ & $120 \pm 30$ & $97 \pm 20$ \\
\hline
\end{tabular}



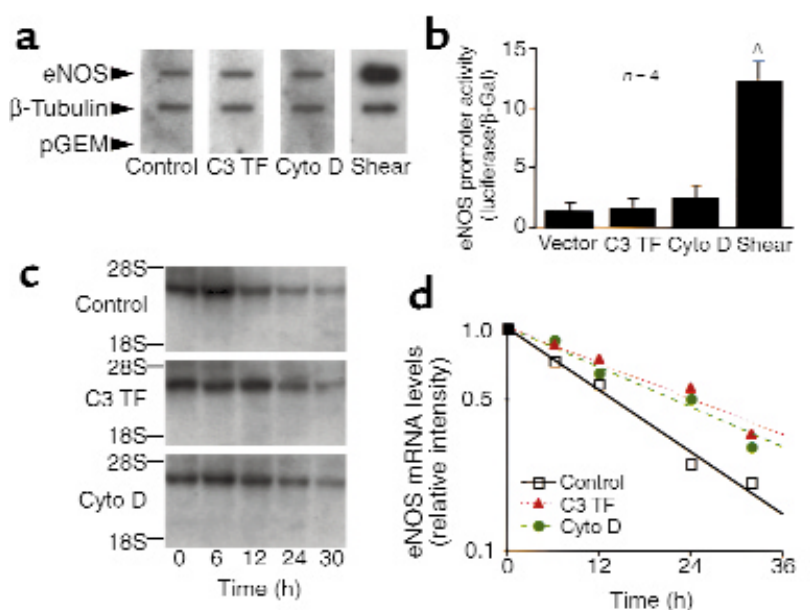

d

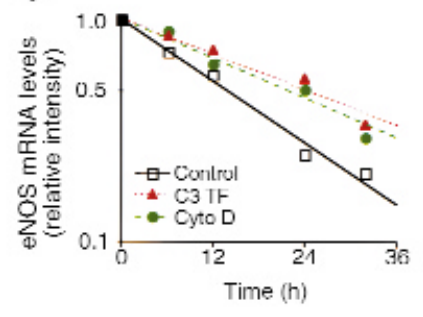

Figure 3

(a) Nuclear run-on and (b) eNOS promoter-luciferase assay showing the effects of C3 TF $(50 \mu \mathrm{g} / \mathrm{mL})$, cyto $\mathrm{D}(1 \mu \mathrm{M})$, and laminar shear stress ( 12 dynes $/ \mathrm{cm}^{2}, 24$ hours) on eNOS gene transcription. Laminar shear stress was applied to endothelial monolayers using a parallel plate chamber apparatus. The $\beta$-tubulin and vector pGEM (Promega Corp., Madison, Wisconsin, USA) cDNA served as controls for loading and nonspecific binding, respectively. The eNOS promoter (-1.8 kb) was linked to a luciferase reporter gene (pGL.2) and transfected into bovine endothelial cells by the Lipofectamine method (GIBCO BRL, Grand Island, New York, USA). The differences between control or vector and laminar shear stress were statistically significant $\left({ }^{A} P<0.05\right)$. (c) Northern and (d) densitometric analyses showing the effect of C3 TF or cyto D on eNOS mRNA half-life. mRNA synthesis was blocked with DRB.

$\mathrm{fmol} / \mathrm{mg} / \mathrm{min}, P<0.05)$, suggesting that eNOS upregulation is specifically mediated by Rho's effect on the actin cytoskeleton and not by potential alternative downstream pathways of Rho. To determine whether genetic increases in the actin cytoskeleton can affect eNOS expression, competitive RT-PCR was performed on aortas from gelsolin ${ }^{-/}$mice and their control littermates (i.e., SV/129-C57BL/6 hybrids) (Figure 6b). Previous studies have shown that fibroblasts in gelsolin ${ }^{-/-}$mice have increased Rac and actin stress fibers $(21,22)$. Using $10^{-2}$ atmol of mutant eNOS to compete with reverse-transcribed mRNA from the samples, we found that eNOS mRNA in aortas from gelsolin ${ }^{-/-}$mice was decreased by $22 \pm 2 \%$ compared with that of littermates $(P<0.05, n=$ 3). These findings indicate that genetic increases in actin stress fiber formation are associated with a decrease in eNOS expression, suggesting that the endothelial actin cytoskeleton and eNOS expression are inversely related.

Regulation of cerebral blood flow by statins. Since cerebral vascular tone is regulated by endothelium-derived NO, we investigated whether increased eNOS activity produced by changes in the actin cytoskeleton leads to increased cerebral blood flow. We have previously shown that statins upregulate eNOS and increase cerebral blood flow in wild-type but not $e \mathrm{NOS}^{-/-}$mice (9). However, it is not known whether statins can augment cerebral blood flow to an ischemic territory of the brain following cerebrovascular occlusion. Therefore, region- al variations in cerebral blood flow, were measured in mice treated with simvastatin using $\left[{ }^{14} \mathrm{C}\right]$-iodoantipyrine autoradiography. The brighter red-yellow colors correspond to higher flows, while the darker bluepurple colors correspond to lower flows. Following MCA occlusion, there was little or no blood flow to the core infarct zone of the parietal lobe (Figure 7a). The blood flow in the adjacent peri-ischemic region, or "penumbra," was slightly higher than that of the core infarct zone, but this was, nevertheless, substantially lower than that of the nonischemic contralateral hemisphere. In mice treated with simvastatin, the entire core infarct zone was smaller, and within the core infarct zone and the penumbra, the blood flow was substan-
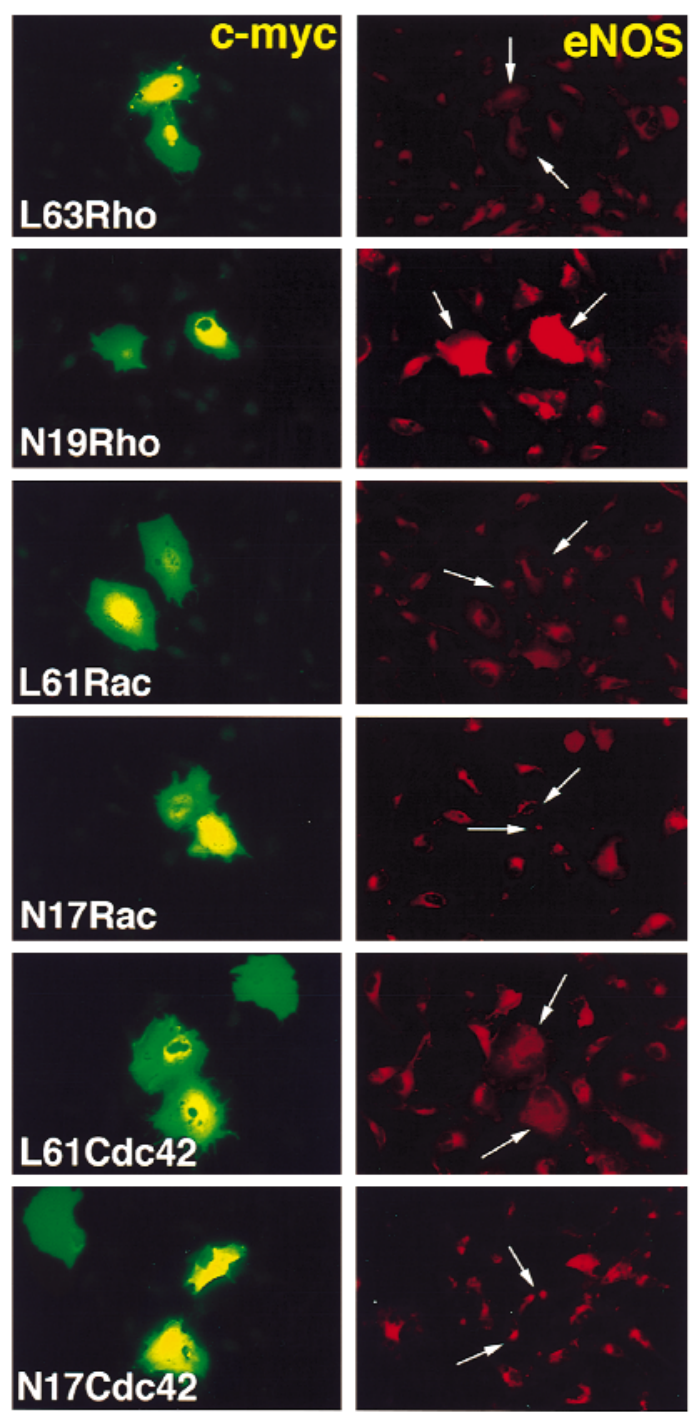

Figure 4

Upregulation of eNOS by inhibition of Rho. Double immunofluorescence showing the effects of constitutively active $(L)$ or dominant-negative (N) c-myc-tagged Rho constructs RhoA, Rac-1, and Cdc42 on endothelial eNOS expression. Immunofluorescence staining using antibodies to c-myc (FITC, green) and eNOS (TRITC, red) was performed three times with similar results. Cells transfected with the indicated Rho cDNA (i.e., c-myc immunofluorescence) are denoted by arrows. 
a

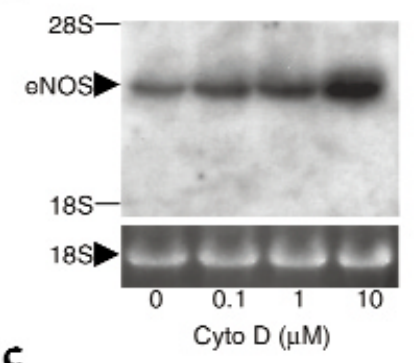

c
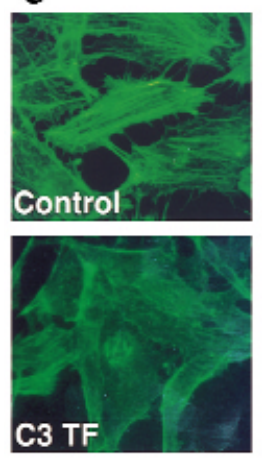
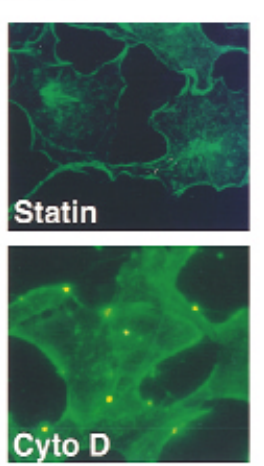

b
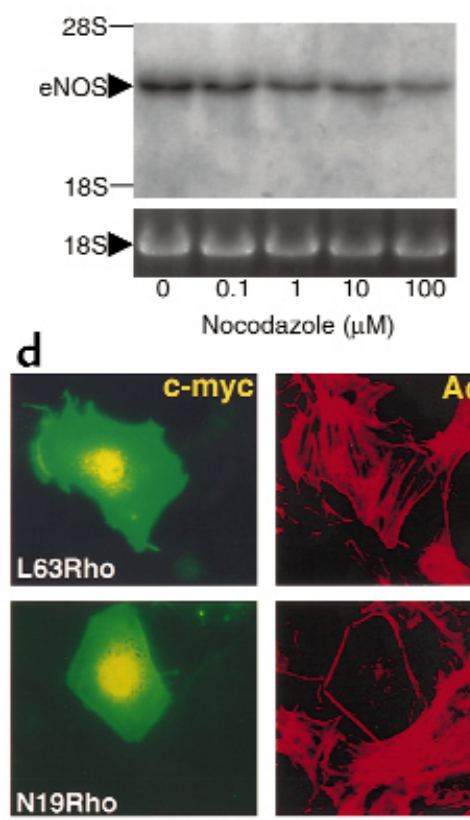

\section{Figure 5}

Northern analyses showing the effects of (a) cyto D and (b) nocodazole on eNOS mRNA levels. (c) Immunofluorescence studies showing the effects of C3 TF $(50 \mu \mathrm{g} / \mathrm{mL})$, cyto D (1 $\mu \mathrm{M})$, and nocodazole $(1 \mu \mathrm{M})$ on endothelial actin stress fibers as determined by phalloidin staining. (d) Double immunofluorescence showing the effects of constitutively active (L63) or dominant-negative (N19) cmyc-tagged RhoA constructs on endothelial actin stress fibers. Immunofluorescence staining was performed using antibodies to c-myc (FITC, green) and phalloidin (TRITC, red). tially higher compared with that of vehicle-treated mice (Figure $7 \mathrm{~b}$ ). These results suggest that inhibition of Rho-mediated endothelial actin cytoskeleton leads to an increase in cerebral blood flow.

Neuroprotection mediated by inbibition of the actin cytoskeleton. To determine whether changes in the endothelial actin cytoskeleton attenuate cerebral ischemic injury, we measured cerebral infarct size following 2 hours of MCA occlusion and 22 hours of reperfusion. In contrast to statins and C3 transferase, cytochalasin D was administered for no more than 24 hours, to avoid potential cytotoxicity. When physiological parameters such as blood pressure, relative cerebral blood flow, blood gases $\left(\mathrm{pH}, \mathrm{PaO}_{2}\right.$, $\mathrm{PaCO}_{2}$ ), and core body temperature were measured, there were no differences between treatment groups except for slightly higher $\mathrm{PaO}_{2}$ in mice treated with cytochalasin D (Table 1).

Treatment with simvastatin, C3 transferase, or cytochalasin D decreased cerebral infarct size by $38 \pm$ $9 \%, 30 \pm 5 \%$, and $41 \pm 7 \%$, respectively, in wild-type $\mathrm{SV} / 129-\mathrm{C} 57 \mathrm{BL} / 6$ mice (littermates for $\mathrm{eNOS}^{-/-}$mice) $(P<0.05$ for C3 transferase and cytochalasin D compared with vehicle) (Figure 8 , a and $b$ ). In mice treated with simvastatin or $\mathrm{C} 3$ transferase, the reduction in cerebral infarct size was also significant when the values were corrected for brain swelling $\left(62 \pm 5 \mathrm{~mm}^{3}\right.$ compared with $88 \pm 4 \mathrm{~mm}^{3}$ for vehicle-treated mice, $P<$ $0.05)$. In mice treated with a lower concentration of cytochalasin D $(0.1 \mathrm{mg} / \mathrm{kg})$, there was no significant increase in eNOS expression or decrease in cerebral infarct size (data not shown). To determine whether eNOS mediates the neuroprotective effects of simvastatin, C3 transferase, or cytochalasin D, we performed similar MCA occlusion studies in $\mathrm{eNOS}^{-/-}$mice. a

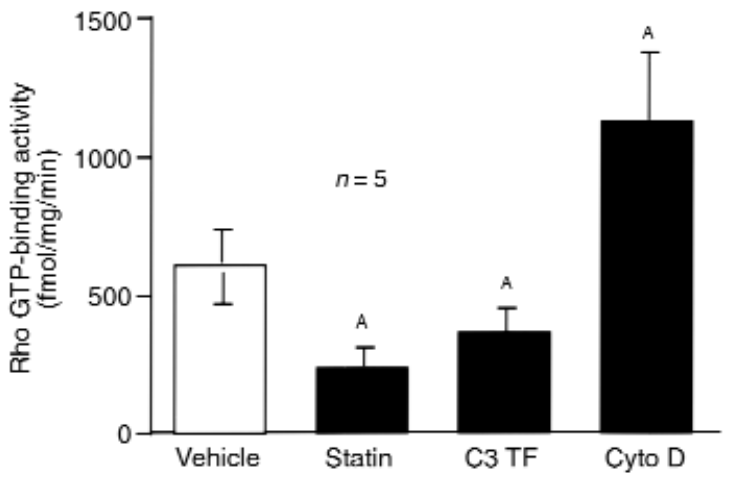

b

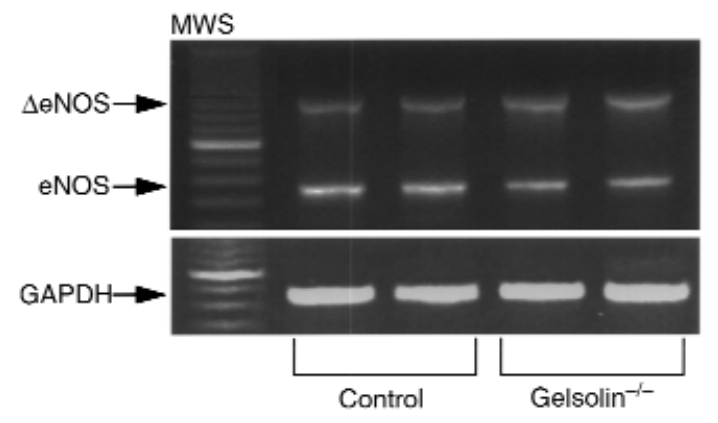

Figure 6

(a) Rho GTP-binding activity was measured in aortas from mice treated with saline (Vehicle), preactivated simvastatin (Statin, 20 $\mathrm{mg} / \mathrm{kg} / \mathrm{d}$ subcutaneously for 14 days), C3 transferase (C3 TF, 10 $\mu \mathrm{g} / \mathrm{d}$ subcutaneously for 14 days), and cytochalasin D (Cyto D, 1 $\mathrm{mg} / \mathrm{kg}$ intraperitoneally for 24 hours). The differences between vehicle-treated mice and mice treated with statin, C3 TF, and cyto D were statistically significant ( $\left.{ }^{A} P<0.05\right)$. (b) Competitive RT-PCR showing the amounts of eNOS mRNA in aortas from gelsolin ${ }^{-/-}$mice and their littermates (Control). For all conditions, $10^{-2}$ atmol of mutant eNOS was used to compete with wild-type eNOS. 

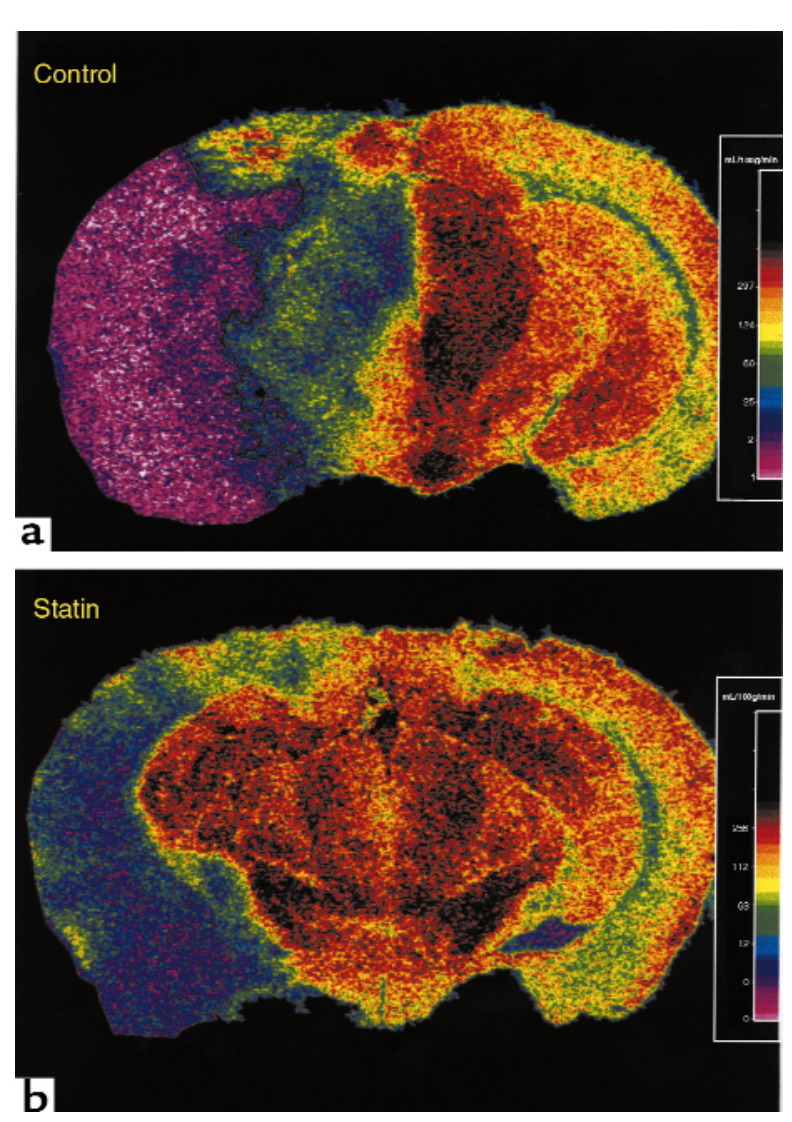

Figure 7

Pseudo-color coronal brain sections (mid-thalamic level) from $\left[{ }^{14} \mathrm{C}\right]-$ iodoantipyrine autoradiography showing regional variations in cerebral blood flow following MCA occlusion in mice treated with vehicle (a) or statin (b). The level of radioactivity is color-coded to reflect higher (yellow-red) and lower (blue-purple) regional blood flow.

Although $e \mathrm{NOS}^{-/-}$mice have larger cerebral infarct size following MCA occlusion compared with that of wildtype mice, treatment with simvastatin, C3 transferase, or cytochalasin $\mathrm{D}$ had absolutely no effect on reducing cerebral infarct size in $\mathrm{eNOS}^{-/-}$mice $(P>0.05)$ (Figure $8 \mathrm{~b})$. These findings suggest that, similar to statins (9), most, if not all, of the neuroprotective effects of these agents are mediated through eNOS.

\section{Discussion}

We have shown that inhibition of Rho-mediated endothelial actin cytoskeleton increases eNOS expression and activity. This leads to increased cerebral blood flow and decreased cerebral infarct size following cerebrovascular occlusion. Most, if not all, of this neuroprotective effect is mediated by eNOS, since $\mathrm{eNOS}^{-/-}$mice do not exhibit any neuroprotection when treated with the Rho inhibitor C3 transferase or the actin cytoskeleton disrupter cytochalasin D. Although Rho can modulate the actin cytoskeleton, inhibition of Rho by itself is not sufficient to upregulate eNOS, since cytochalasin D increases eNOS expression despite compensatory increase in vascular Rho activity. These results are similar to the effects of HMGCoA reductase inhibitors (9) and suggest that signaling pathways that ultimately affect the integrity of the

endothelial actin cytoskeleton increase eNOS expression.

Surprisingly, the mechanism by which the actin cytoskeleton regulates eNOS expression is not due to the induction of eNOS gene transcription but, instead, to the post-transcriptional stabilization of eNOS mRNA. Since hypoxia, oxidized LDL, and cytokines such as TNF- $\alpha$ decrease eNOS expression by reducing eNOS mRNA stability $(17,23-25)$, the ability of Rho and actin cytoskeletal inhibitors to prolong eNOS halflife may make them effective agents in counteracting conditions that downregulate eNOS expression. Indeed, inhibition of Rho geranylgeranylation and the actin cytoskeleton by statins prevents the downregulation of eNOS by oxidized LDL and TNF- $\alpha$ and under hypoxic conditions $(17,23,25)$. Thus, the stability of eNOS mRNA may be an important general mechanism by which eNOS expression is regulated.

The actin cytoskeleton provides the structural framework for cell shape, movement, and polarity (15). Recent studies suggest that the anchoring of mRNAs to the actin cytoskeleton and their colocalization with ribosomes and RNA-binding protein complexes are

a

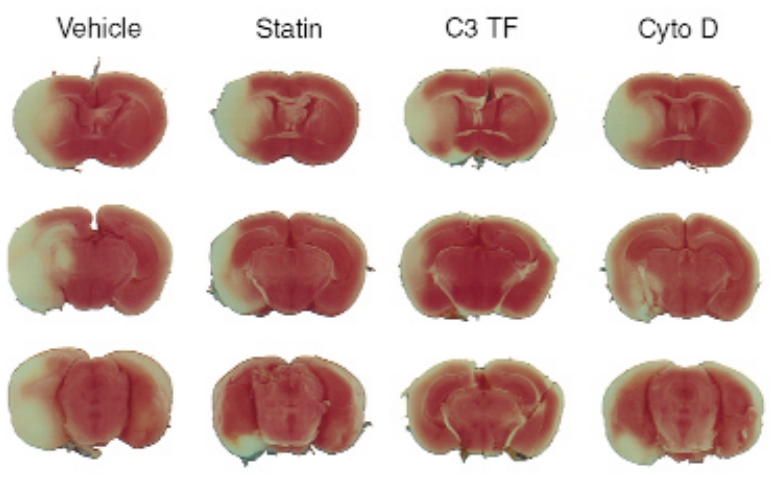

b

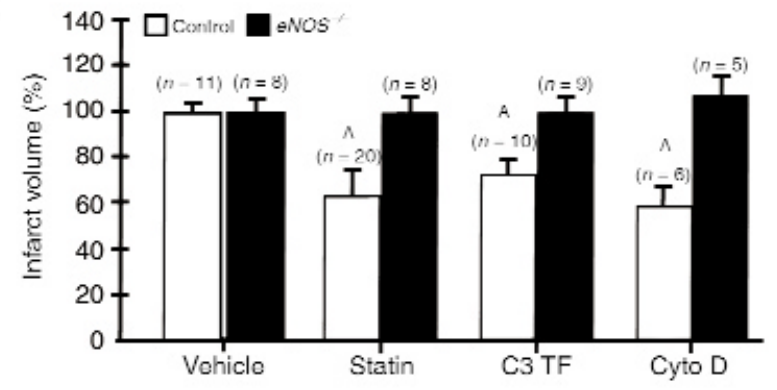

\section{Figure 8}

(a) Coronal brain sections (top to bottom, rostral to caudal) stained with 2,3,5-triphenyltetrazolium chloride in wild-type SV/129C57BL/ 6 mice treated with vehicle, simvastatin (Statin, $20 \mathrm{mg} / \mathrm{kg} / \mathrm{d}$ subcutaneously for 14 days), C3 TF ( $10 \mu \mathrm{g} / \mathrm{d}$ subcutaneously for 14 days), and cyto D ( $1 \mathrm{mg} / \mathrm{kg}$ intraperitoneally for 24 hours) before undergoing MCA occlusion and reperfusion. (b) Cerebral infarct size (Infarct volume) following MCA occlusion and reperfusion in wildtype SV/129-C57BL/ 6 littermates (Control) and eNOS ${ }^{-/-}$mice treated with $\mathrm{C} 3 \mathrm{TF}$ or cyto $\mathrm{D}$. The values for the infarct volume are relative to those of vehicle-treated mice. The differences between vehicle and treatment conditions were statistically significant $\left({ }^{A} P<0.05\right)$ in wild-type littermates, but not in $\mathrm{NOS}^{-1-}$ mice. 
necessary for their stability and translational expression (26). Thus, Rho-controlled reorganization of the actin cytoskeleton may play a key role in the movement and subcellular localization of specific mRNAs $(27,28)$. For example, the peripheral localization of $\beta$-actin mRNA is required for the assembly of contractile and motility apparatus at the leading edge of moving cells (29). Thus, Rho may affect eNOS mRNA stability, in part, by regulating the cytoskeletal localization of eNOS mRNA. The precise signaling pathway by which Rho mediates the assembly of the focal adhesion complex and reorganization of the cytoskeleton, however, is still poorly understood $(15,19,30)$.

Rho binds and activates the serine-threonine p164 Rho-associated kinase and its close relative, p160 Rhoassociated coiled-coil-containing protein kinase (31-33). A primary downstream effect of Rho kinase is the phosphorylation of MLC, which is required for the formation of actin stress fibers and focal adhesion complexes $(33,34)$. Although there are many other downstream targets of Rho kinase, such as protein kinase $\mathrm{N}(35,36)$, phosphatidylinositol 4-phosphate5 kinase (PIP-5) (37), and the actin-binding proteins ezrin/radixin/moesin (32), our findings with cytochalasin D suggest that Rho-induced MLC phosphorylation, focal adhesion assembly, and actin cytoskeletal reorganization mediate the upregulation of eNOS expression. Thus, modulation of the endothelial actin cytoskeleton may represent new potential targets for vascular pharmacotherapy.

Chronic treatment with statins elevates cerebral blood flow by eNOS upregulation and NO generation $(9,38)$. The upregulation of neuronal NOS or inducible NOS is not observed. The statin flow effect is enhanced by infusing the eNOS substrate L-arginine, but not in $e \mathrm{NOS}^{-/-}$mice. Consistent with these flow observations, L-arginine provides enhanced neuroprotection when combined with statins (data not shown). We believe that improved collateral flow and microcirculation explain the findings, as flow is enhanced in both core and penumbra of the ischemic territory. Improved collateral flow and microcirculation may also reflect antiplatelet and antileukocyte actions of $\mathrm{NO}(4,6)$. For these reasons, the statins and more specific drugs targeting the endothelial actin cytoskeleton may prove beneficial in cerebral ischemia and in other conditions characterized by low cerebral blood flow.

In summary, our findings that gene expression is regulated by changes in the endothelial actin cytoskeleton may establish the mechanistic basis for some of the noncholesterol effects of statins on bone (39) and the cardiovascular system (40-43). The absence of neuroprotection in $\mathrm{NNOS}^{-/-}$mice emphasizes the importance of endothelium-derived NO in augmenting cerebral blood flow and, potentially, in limiting the impact of platelet and white blood cell accumulation on tissue viability following ischemia and reperfusion. We propose that agents that modulate the endothelial actin cytoskeleton may be beneficial for treating ischemic stroke.

\section{Acknowledgments}

We thank Christian Waeber for technical assistance; Alan Hall (University of London, United Kingdom) and Wouter Moolenaar (The Netherlands Cancer Institute) for Rho, Rac-1, and Cdc42 cDNA mutants; and David Kwiatkowski (Brigham and Women's Hospital) for gelsolin $^{-/-}$mice and littermates. This work was supported by grants from the National Institutes of Health (HL52233, HL-26202, and NS-10828), the American Heart Association, and the Deutsche Forschungsgemeinschaft (La1057/1-1 and En343/6-1).

1. Harrison, D.G. 1997. Cellular and molecular mechanisms of endothelial cell dysfunction. J. Clin. Invest. 100:2153-2157.

2. Huang, Z., et al. 1996. Enlarged infarcts in endothelial nitric oxide synthase knockout mice are attenuated by nitro-L-arginine. J. Cereb. Blood Flow Metab. 16:981-987.

3. Furchgott, R.F., and Zawadzki, J.V. 1980. The obligatory role of endothelial cells in the relaxation of arterial smooth muscle by acetylcholine. Nature. 288:373-376.

4. Radomski, M.W., Palmer, R.M., and Moncada, S. 1990. An L-arginine/nitric oxide pathway present in human platelets regulates aggregation. Proc. Natl. Acad. Sci. USA. 87:5193-5197.

5. Rudic, R.D., et al. 1998. Direct evidence for the importance of endothelium-derived nitric oxide in vascular remodeling. J. Clin. Invest. 101:731-736.

6. Kubes, P., Suzuki, M., and Granger, D.N. 1991. Nitric oxide: an endogenous modulator of leukocyte adhesion. Proc. Natl. Acad. Sci. USA. 88:4651-4655.

7. Huang, P.L., et al. 1995. Hypertension in mice lacking the gene for endothelial nitric oxide synthase. Nature. 377:239-242.

8. Yamamoto, S., Golanov, E.V., Berger, S.B., and Reis, D.J. 1992. Inhibition of nitric oxide synthesis increases focal ischemic infarction in rat.J. Cereb. Blood Flow Metab. 12:717-726.

9. Endres, M., et al. 1998. Stroke protection by 3-hydroxy-3-methylglutaryl (HMG)-CoA reductase inhibitors mediated by endothelial nitric oxide synthase. Proc. Natl. Acad. Sci. USA. 95:8880-8885.

10. Morikawa, E., Huang, Z., and Moskowitz, M.A. 1992. L-arginine decreases infarct size caused by middle cerebral arterial occlusion in SHR. Am. J. Physiol. 263:H1632-H1635.

11. Morikawa, E., et al. 1994. L-arginine infusion promotes nitric oxidedependent vasodilation, increases regional cerebral blood flow, and reduces infarction volume in the rat. Stroke. 25:429-435.

12. Wang, Q., Patton, W.F., Hechtman, H.B., and Shepro, D. 1997. A novel anti-inflammatory peptide inhibits endothelial cell cytoskeletal rearrangement, nitric oxide synthase translocation, and paracellular permeability increases. J. Cell Physiol. 172:171-182.

13. Levin, E.R., et al. 1998. Role of vascular endothelial cell growth factor in Ovarian Hyperstimulation Syndrome. J. Clin. Invest. 102:1978-1985.

14. Knudsen, H.L., and Frangos, J.A. 1997. Role of cytoskeleton in shear stress-induced endothelial nitric oxide production. Am. J. Physiol. 273:H347-H355.

15. Hall, A. 1998. Rho GTPases and the actin cytoskeleton. Science. 279:509-514.

16. Laufs, U., and Liao, J.K. 1998. Post-transcriptional regulation of endothelial nitric oxide synthase mRNA stability by Rho GTPase. J. Biol. Chem. 273:24266-24271.

17. Laufs, U., LaFata, V., Plutzky, J., and Liao, J.K. 1998. Upregulation of endothelial nitric oxide synthase by HMG CoA reductase inhibitors. Circulation. 97:1129-1135.

18. Huang, Z., et al. 1994. Effects of cerebral ischemia in mice deficient in neuronal nitric oxide synthase. Science. 265:1883-1885.

19. Van Aelst, L., and D'Souza-Schorey, C. 1997. Rho GTPases and signaling networks. Genes Dev. 11:2295-2322.

20. Enomoto, T. 1996. Microtubule disruption induces the formation of actin stress fibers and focal adhesions in cultured cells: possible involvement of the rho signal cascade. Cell Struct. Funct. 21:317-326.

21. Witke, W., et al. 1995. Hemostatic, inflammatory, and fibroblast responses are blunted in mice lacking gelsolin. Cell. 81:41-51.

22. Azuma, T., Witke, W., Stossel, T.P., Hartwig, J.H., and Kwiatkowski, D.J. 1998. Gelsolin is a downstream effector of rac for fibroblast motility. EMBO J. 17:1362-1370.

23. Laufs, U., Fata, V.L., and Liao, J.K. 1997. Inhibition of 3-hydroxy-3methylglutaryl (HMG)-CoA reductase blocks hypoxia-mediated downregulation of endothelial nitric oxide synthase. J. Biol. Chem. 272:31725-31729.

24. Hernandez-Perera, O., et al. 1998. Effects of the 3-hydroxy-3-methylglu- 
taryl-CoA reductase inhibitors, atorvastatin and simvastatin, on the expression of endothelin-1 and endothelial nitric oxide synthase in vascular endothelial cells. J. Clin. Invest. 101:2711-2719.

25. Yoshizumi, M., Perrella, M.A., Burnett, J.C., Jr., and Lee, M.E. 1993. Tumor necrosis factor downregulates an endothelial nitric oxide synthase mRNA by shortening its half-life. Circ. Res. 73:205-209.

26. Bassell, G., and Singer, R.H. 1997. mRNA and cytoskeletal filaments. Curr. Opin. Cell Biol. 9:109-115.

27. Chicurel, M.E., Singer, R.H., Meyer, C.J., and Ingber, D.E. 1998. Integrin binding and mechanical tension induce movement of mRNA and ribosomes to focal adhesions. Nature. 392:730-733.

28. Nasmyth, K., and Jansen, R.P. 1997. The cytoskeleton in mRNA localization and cell differentiation. Curr. Opin. Cell Biol. 9:396-400.

29. Bassell, G.J., et al. 1998. Sorting of beta-actin mRNA and protein to neurites and growth cones in culture. J. Neurosci. 18:251-265.

30. Hall, A. 1994. Small GTP-binding proteins and the regulation of the actin cytoskeleton. Annu. Rev. Cell Biol. 10:31-54.

31. Matsui, T., et al. 1996. Rho-associated kinase, a novel serine/threonine kinase, as a putative target for small GTP binding protein Rho. EMBOJ. 15:2208-2216.

32. Matsui, T., et al. 1998. Rho-kinase phosphorylates COOH-terminal threonines of ezrin/radixin/moesin (ERM) proteins and regulates their headto-tail association. J. Cell Biol. 140:647-657.

33. Ishizaki, T., et al. 1996. The small GTP-binding protein Rho binds to and activates a $160 \mathrm{kDa}$ Ser/Thr protein kinase homologous to myotonic dystrophy kinase. EMBO J. 15:1885-1893.

34. Chrzanowska-Wodnicka, M., and Burridge, K. 1996. Rho-stimulated contractility drives the formation of stress fibers and focal adhesions. $J$. Cell Biol. 133:1403-1415.

35. Amano, M., et al. 1996. Identification of a putative target for Rho as the serine-threonine kinase protein kinase N. Science. 271:648-650.

36. Watanabe, G., et al. 1996. Protein kinase N (PKN) and PKN-related protein rhophilin as targets of small GTPase Rho. Science. 271:645-648.

37. Chong, L.D., Traynor-Kaplan, A., Bokoch, G.M., and Schwartz, M.A. 1994. The small GTP-binding protein Rho regulates a phosphatidylinositol 4-phosphate 5-kinase in mammalian cells. Cell. 79:507-513.

38. Yamada, M., et al. 2000. Endothelial nitric oxide synthase-dependent cerebral blood flow augmentation by L-arginine after chronic statin treatment. J. Cereb. Blood Flow Metab. 20:709-717.

39. Mundy, G., et al. 1999. Stimulation of bone formation in vitro and in rodents by statins. Science. 286:1946-1949.

40. Laufs, U., Marra, D., Node, K., and Liao, J.K. 1999. 3-Hydroxy-3-methylglutaryl-CoA reductase inhibitors attenuate vascular smooth muscle proliferation by preventing rho GTPase-induced down-regulation of p27(Kip1). J. Biol. Chem. 274:21926-21931.

41. Wojciak-Stothard, B., Williams, L., and Ridley, A.J. 1999. Monocyte adhesion and spreading on human endothelial cells is dependent on Rho-regulated receptor clustering. J. Cell Biol. 145:1293-1307.

42. Essig, M., et al. 1998. 3-Hydroxy-3-methylglutaryl coenzyme A reductase inhibitors increase fibrinolytic activity in rat aortic endothelial cells. Role of geranylgeranylation and Rho proteins. Circ. Res. 83:683-690.

43. Aoki, H., Izumo, S., and Sadoshima, J. 1998. Angiotensin II activates RhoA in cardiac myocytes: a critical role of RhoA in angiotensin IIinduced premyofibril formation. Circ. Res. 82:666-676. 